\title{
Using the health action process approach to predict and improve health outcomes in individuals with type 2 diabetes mellitus
}

This article was published in the following Dove Press journal:

Diabetes, Metabolic Syndrome and Obesity:Targets and Therapy

16 October 2014

Number of times this article has been viewed

\section{Mariana MacPhail' \\ Barbara Mullan ${ }^{2}$ \\ Louise Sharpe' \\ Carolyn MacCann' \\ Jemma Todd'}

'School of Psychology, University of Sydney, Sydney, NSW, Australia; ${ }^{2}$ School of Psychology and Speech Pathology, Curtin University, Bentley, WA, Australia
Correspondence: Barbara Mullan School of Psychology and Speech Pathology, Curtin University, Bentley, WA 6102, Australia Tel +6I 892663473 Fax +6I 892662464 Email barbara.mullan@curtin.edu.au
Background: The purpose of this study was to explore the predictive utility of the Health Action Process Approach (HAPA) and test a HAPA-based healthy eating intervention, in adults with type 2 diabetes mellitus.

Materials and methods: The study employed a prospective, randomized, controlled trial design. The 4-month intervention consisted of self-guided HAPA-based workbooks in addition to two telephone calls to assist participants with the program implementation, and was compared to "treatment as usual". Participants $(n=87)$ completed health measures (diet, body mass index [BMI], waist circumference, blood pressure, blood glucose levels, lipid levels, and diabetes distress) and HAPA measures prior to the intervention and again upon completion 4 months later.

Results: The overall HAPA model predicted BMI, although only risk awareness and recovery self-efficacy were significant independent contributors. Risk awareness, intentions, and selfefficacy were also independent predictors of health outcomes; however, the HAPA did not predict healthy eating. No significant time $\times$ condition interaction effects were found for diet or any HAPA outcome measures.

Conclusion: Despite the success of HAPA in predicting health outcomes for those with type 2 diabetes mellitus, the intervention was unsuccessful in changing healthy eating or any of the other measured variables, and alternative low-cost health interventions for those with type 2 diabetes mellitus should be explored.

Keywords: intervention, healthy eating, theory, risk awareness, self-efficacy

\section{Introduction}

Diabetes is associated with shortened life expectancy and reduced quality of life. ${ }^{1}$ Over a 15-year period, the proportion of people in Australia with diagnosed diabetes more than doubled, from $1.3 \%$ to $3.6 \%$, and the rise is largely driven by an increase in the prevalence of type 2 diabetes mellitus (T2D). ${ }^{2}$ The Australian Diabetes, Lifestyle and Obesity study $2004-2005^{2}$ estimated that every year, $0.8 \%$ of adults develop diabetes (275 adults a day). Globally, $2.8 \%$ of the population was estimated to have diabetes in 2000 , which was predicted to rise to $4.4 \%$ by $2030 .{ }^{3}$ The increase in the incidence of T2D has been associated with aging populations and an increase in rates of excess weight, unhealthy nutrition, and inactive lifestyles. ${ }^{4}$

The risk of developing T2D increases with higher levels of body mass index (BMI). ${ }^{5}$ This relationship between being overweight and T2D is not surprising considering that those who are overweight are less able to process insulin effectively. ${ }^{1}$ Healthy eating can improve T2D control and reduce complications, such as cardiovascular 
disease. ${ }^{6}$ Whether for weight loss or maintenance, healthy eating can assist with achieving and maintaining blood glucose, blood pressure, and lipid levels in the target ranges (or as close to target as possible). However, improving and maintaining healthy eating over time is challenging, ${ }^{1,4,7}$ and an understanding of the processes involved in this is critical to encouraging health behavior change and thus, ultimately, for improving health. ${ }^{8}$

One model that has been used to explain health behavior engagement is the Health Action Process Approach (HAPA). ${ }^{9}$ The HAPA proposes that engaging in healthy behavior consists of two processes: forming an intention (motivation phase), followed by a stage of planning to act and action (volition phase). In the motivational phase, risk awareness, outcome expectancies, and task self-efficacy lead to the formation of an intention to either adopt a health protective behavior or change a health risk behavior. Risk awareness refers to the perceived health threat or concern that is needed to mobilize action. Task self-efficacy is the degree to which a person believes he or she is able to complete a particular behavior. ${ }^{10,11}$ Outcome expectancies refer to whether an individual believes the behavior will bring about the desired change. If the evaluation of the outcome of the behavior is positive, an individual is more likely to form an intention to engage in this behavior.

The volitional phase within the HAPA is comprised of action planning, coping planning, coping self-efficacy, and recovery self-efficacy. These components reduce impulsive and trial-and-error action that results in ineffective allocation of resources. ${ }^{11}$ Planning operates similarly to implementation intentions, in that plans commit the individual to a specific course of action when certain environmental conditions are present. ${ }^{12}$ For the process of maintaining behavior, coping self-efficacy helps to overcome incompatible goals, urges, interfering emotions, and environmental obstacles that occur during the execution of the planned behavior. Finally, recovery self-efficacy allows an individual to recover if they experience behavior relapses or they disengage from the planned action. Those with higher self-efficacy are expected to envisage more positive opportunities to act, have more elaborate plans, persevere more, and recover from setbacks more readily. ${ }^{9}$

There is support for use of the HAPA in predicting healthy eating in the general (nonclinical) population. In two studies that used the HAPA to predict low-fat, high-fiber, and high-vitamin diet, intention and coping self-efficacy explained $33 \%$ to $48 \%$ of the variance in nutrition up to 6 months later. ${ }^{13,14}$ The HAPA has also been recently applied to an obese sample, and partial support for the model predicting physical activity was found within this group. ${ }^{15}$ There was also recent support for the HAPA in predicting physical activity in individuals with T2D. ${ }^{16}$ Across a large sample of individuals with T2D, support was found for the HAPA principles. Specifically, there was evidence of differences between the motivation and volitional stages of health behavior change, and of differences between the intentional and action stages within the volition stage. However, this study did not include dietary behaviors, which are yet to be explored within a HAPA framework for this population.

Research has found evidence suggesting that self-management programs for chronic diseases, including diabetes, can improve health status at 6 months. ${ }^{17}$ For example, a HAPA-based physical activity planning intervention for obese individuals, consisting of brief counseling and telephone sessions, was found to improve physical activity up to 12 months later, although weight changes were not found. ${ }^{18}$ While the HAPA has not been directly applied to individuals with T2D, the HAPA has been used to design and investigate interventions to reduce risk of diabetes. In one intervention targeting individuals at risk of T2D, ${ }^{19}$ improvements in waist circumference, BMI, total cholesterol and low-density lipoprotein (LDL) cholesterol were found at 3 months, and improvements in weight, waist circumference, glucose, lipids, blood pressure, and psychological distress were also found at a 12-month follow-up visit.

In an attempt to improve the accessibility and effectiveness of weight-management interventions, new delivery approaches, such as telephone calls, mail, and e-mail, have been trialed. In a systematic review of self-management interventions for chronic disease, Warsi et $\mathrm{al}^{20}$ found that intervention outcomes were not dependent on delivery method, suggesting that alternatives to face-to-face treatment, such as written and telephone-based modes, may be an effective means of delivering low-cost, easily accessible programs. For example, a randomized controlled trial in which mail and phone support was given as an adjunct to weight loss interventions in an overweight sample was shown to be effective at reducing weight at 6 months, although this difference was not maintained at 12 months. ${ }^{21}$ In an online diabetes self-management program, Lorig et $\mathrm{al}^{29}$ found mixed results; however, those who participated in the intervention had improved blood glucose levels 6 months later. Given that theory-based interventions tend to be more successful generally, and specifically in diabetes, ${ }^{22}$ than those not based on theory, ${ }^{17}$ it may be that the effectiveness of alternative non-face-to-face treatments can be improved by the use of theories such as the HAPA. 


\section{Aims}

To date there has been little research exploring the relationship between HAPA variables and health behaviors in individuals with T2D; there have also not been any HAPA-based interventions in which alternative delivery approaches were used to target these behaviors in individuals with T2D. Therefore, the purpose of this research was 1) to determine whether HAPA variables were associated with diabetes-related health outcomes and dietary behavior, and 2) to determine the effectiveness of a self-directed HAPA-based "healthy eating" intervention in bringing about changes in HAPA variables, dietary behavior, and health outcomes. The health outcomes of interest were BMI and waist circumference, glycemic control, blood pressure, lipid levels, and diabetes emotional distress.

\section{Materials and methods Participants}

A total of 146 individuals attending primary care diabetes clinics in Australia were identified by their treating general practitioners (GPs) as having T2D and were approached to participate in this study. In all, 87 individuals provided consent (response rate $=60 \%$ ). The exclusion criteria were a diagnosis of type 1 or gestational diabetes (ie, not T2D); not proficient in English; or having acute psychosis or dementia; however, none of those who provided consent were excluded. Finally, 77 participants completed the study (retention rate $=89 \%$ ); three participants withdrew prior to completing the baseline measures, and seven individuals did not complete the follow up 4 months later. This study was approved by the University of Sydney's Human Research Ethics Committee. All participants gave written consent.

\section{Measures}

The questionnaires at baseline and follow up were identical, with the exception of demographics, which were collected at baseline only. The items used to measure the HAPA model ${ }^{23}$ were adapted from those used in previous studies of dietary behavior and physical activity. ${ }^{13,14,23,24}$ The items assessed risk awareness (nine items, eg, "If you continue with your current eating pattern, how likely is it that you will be less physically healthy"); outcome expectancies (three items, eg, "How true is it that if I am able to improve and maintain healthier eating patterns I will be more physically healthy"); task self-efficacy (six items, eg, "I am confident that I am able to improve my eating pattern, even if I have to make a detailed plan to have appropriate food available"); intention (one item: "How true is it that I intend to improve my eating pattern"); planning (a combination of action planning and coping planning) (eleven items, eg, "Over the previous few weeks, I have had my own plan regarding when I will eat healthy food"); coping self-efficacy (seven items, eg, "I am confident that I am able to maintain my eating pattern, even if I have to make and review a detailed plan about what food to have and when"); and recovery self-efficacy (four items, eg, "I am confident that I am able to return to my intended eating pattern, even if I give it up for a meal"). The internal consistency of these variables was high (Cronbach's $\alpha \geq 0.89$ ).

Healthy eating behavior was measured using the Diet Guidelines Index (DGI). ${ }^{25}$ The DGI is a food-based dietary index that measures adherence to healthy eating recommendations over the previous month. It consists of 15 items that reflect current dietary guidelines, ${ }^{26}$ including consumption of vegetable and legumes, fruit, total cereals, meat, total dairy, beverages, sodium, saturated fat, alcoholic beverages, and added sugars. A diet quality score is obtained by summing the indicators of wholegrain cereals, lean meat, low-fat dairy, and dietary variety, and is informed by age- and sex-specific recommendations. Scores range from 0 to 150 , with higher scores representing higher levels of healthy eating. Serving portions are described in the questionnaire, and participants were directed to refer to the healthy eating guidelines ${ }^{26}$ provided for further details. For instance regarding fruit consumption, individuals were required to complete the following statement: "I eat ... servings of fruit per day". The internal consistency was acceptable, Cronbach's $\alpha=0.61$.

Health-related emotional distress was assessed with the Diabetes Distress Scale, ${ }^{27}$ which is a validated measure of diabetes emotional distress, assessing the general emotional burden of diabetes, distress and problems related to treatment, food choices, and social support. The 17 items (eg, "Feeling that diabetes is taking up too much of my mental and physical energy every day") are scored on a six-point scale yielding a sum score (17-102), with higher scores representing greater distress. A previous study found strong internal reliability $(\alpha=0.87),{ }^{27}$ and the internal consistency in the current study was high (Cronbach's $\alpha=0.88$ ).

During a baseline clinic visit, objective physical measurements were obtained by either the diabetes educator or GP; comparable measures were also taken at the 4-month check-up visit. These included weight and height, used to calculate BMI $\left(\mathrm{kg} / \mathrm{m}^{2}\right)$; waist circumference $(\mathrm{cm})$; systolic and diastolic blood pressure $(\mathrm{mm} / \mathrm{Hg})$; lipids (total cholesterol, LDL ["bad" cholesterol], high-density lipoprotein [HDL] ["good" cholesterol], and triglycerides) $(\mathrm{mmol} / \mathrm{L})$; and glycemic control (glycated hemoglobin $\left.\left[\mathrm{HbA}_{1 \mathrm{c}}\right]\right)(\mathrm{mmol} / \mathrm{L}$, with conversions to $\%$ and $\mathrm{mmol} / \mathrm{mol}$ ). 


\section{Intervention}

The intervention was designed to be consistent with the recommendations from the National Collaborating Centre for Chronic Conditions ${ }^{6}$ and the National Institute for Health and Clinical Excellence. ${ }^{28}$ Accordingly, the intervention was theory driven, sustainable, easy to access, integrated into the patients' current management, and tailored to fit their personal needs. The primary components of the intervention were completion of a theory-based workbook ("Ready, Set, Go"), and two telephone calls.

The "Ready, Set, Go" workbook was designed for the current study and was based on the HAPA ${ }^{23}$ and on effective interventions for diabetes and prediabetes self-management that have used elements of the HAPA, ${ }^{23}$ such as self-efficacy and action planning. ${ }^{19,29,30}$ The purpose of the workbook was to provide a simple framework to improve self-efficacy and to support selection, planning, and implementation of healthy eating goals more effectively.

Multiple copies of the workbook were provided for use over the 4 months, between clinic visits. Each copy included four weekly diaries. By using a fresh workbook, participants had the opportunity to reset their goals several times during the study. This allowed a self-monitoring and reevaluation cycle consistent with the HAPA. ${ }^{23}$

The workbook consisted of three modules. "Step One: READY" included behavior change techniques (eg, prompt specific goal setting, prompt practice [imaginary], prompt self-talk) designed to prepare participants for goal selection by informing them of the risks of nonaction, and prompting them to consider likely outcome expectancies of changing their eating behavior, as well as to build task self-efficacy. The primary task in this section of the workbook was a decisional balance sheet, which prompted participants to identify the costs and benefits of eating healthily versus not changing. Participants were subsequently encouraged to enact their behavioral intentions through their reading of the workbook strategies, such as mastery, vicarious experience, verbal persuasion, and emotional arousal (all designed to increase their confidence in doing so).

The aim of "Step Two: SET" was to encourage participants to set specific behavioral goals, based on the Australian Healthy Eating Guidelines, for the following 4 weeks. In contrast to the first module, which focused on the motivational phases of the HAPA model, this module focused on the volitional phase of the model. Specifically, the module included behavior change techniques to target the model predictors of intention, planning, coping self-efficacy, and recovery self-efficacy - for example, participants were required to set
SMART goals (specific, measureable, achievable, relevant, time-bound) in relation to their healthy eating intentions and to complete an action and maintenance plan based on an implementation intention framework. ${ }^{12}$ In addition, they were prompted to anticipate likely barriers and to plan their coping to overcome these (eg, attention control, management of negative emotions, coping with failure, and reinterpreting failure).

The final module, "Step Three: GO", was based on the action phase of the HAPA model and included strategies relevant to the initiation, maintenance, and recovery process of enacting a particular goal (eg, prompt practice, prompt self-monitoring, prompt feedback on performance, agree to a behavioral contract). For example, participants were required to rehearse their new behavior of healthy eating and to monitor and review their progress in achieving their previously set goals in a real-life context. Specifically, participants were encouraged to engage in daily scoring of their level of goal achievement and level of confidence or self-efficacy in doing so, as well as to continue reflecting on their successes and identifying weaknesses in their approach and the usefulness of the strategies they were using to work on goals and overcome barriers. Each copy of the workbook included a weekly diary for participants to record this information. Full details of the intervention workbook can be obtained by contacting the authors.

Although no data was available on participants' actual use of the workbook (eg, whether all modules were completed and amount of time spent using the workbook) due to the self-directed nature of the intervention, the following data on user satisfaction supports the acceptability of the workbook as an intervention tool: $73 \%$ of participants who completed the intervention found the information in the workbook either helpful or very helpful; $80 \%$ found the layout of the workbook helpful or very helpful; $80 \%$ found the phone calls helpful or very helpful; $90 \%$ found the information easy or very easy to understand; $93 \%$ found the process easy or very easy to understand; and $88 \%$ endorsed finding the information and layout enjoyable or very enjoyable.

Telephone calls were used to support patients in the use of the workbooks and to provide any clarification required. The calls assessed the rate of progress and identified areas for improvement and any difficulties participants may have been experiencing with the workbook or their goal setting/initiation. The approach was based on motivational interviewing techniques suggested for health behavior change. ${ }^{31}$ The calls encouraged self-efficacy and planning, 
identification of possible difficulties, goal monitoring, and setting of reinforcements and implementation intention strategies.

\section{Procedure}

Eligible participants received an envelope enclosing an invitation letter from their GP, participant information statement and consent form, and the baseline questionnaire. Participants completed the baseline questionnaire either at home or in consultation with the researcher at the clinic.

Participants were then randomly allocated, via a random numbers table, to complete the intervention or continue with "treatment as usual" (control group). Treatment as usual involved being seen by the GP and/or the diabetes education nurse for assessment and provision of information and advice on how to manage diabetes. These appointments were attended at each diabetes clinic. Participants in the intervention group continued to receive treatment as usual and were additionally provided with the intervention materials (ie, workbook and phone calls). Treatment group allocation was concealed until after participants had completed the assessment. Treating doctors and nurses remained blind to the allocation of participants to groups; however, due to the nature of the study, the researcher was not blinded to the treatment type. Upon attending the diabetes clinic, those in the intervention group were given a workbook ("Ready, Set, Go") and the Australian Healthy Eating Guidelines, ${ }^{30}$ to be used independently by the participants. The intervention also included two brief telephone contacts with the researcher; the first generally occurring within 2 weeks of attending the clinic, and the second, 4 weeks after the first. This approach was designed to integrate the intervention and the patients' general health care in a way that was practical and related to their real-life context. Those in the control group did not receive the intervention materials or phone telephone calls but received the baseline and follow-up measures.

A week prior to their 4-month routine follow-up appointment at the clinic, all participants were mailed a follow-up questionnaire, as well as a \$20 supermarket food voucher. Biological measures of weight, height, waist circumference, blood pressure, and blood glucose levels were collected in the follow-up appointments.

\section{Statistical analyses}

The first aim of the study was to determine whether HAPA variables were associated with diabetes-related health outcomes and dietary behavior. This aim was tested firstly using Pearson's product-moment correlations between healthy eating variables and baseline HAPA variables: risk awareness (relative, absolute, and severity); expectancy; intention; planning (action and coping); and selfefficacy (task, coping and recovery). Secondly, a series of hierarchical linear regressions were conducted to explore whether HAPA variables predicted later health outcomes. Analyses were conducted separately for the following dependent variables: diabetes-related distress; healthy eating; BMI; waist circumference; glycemic control; total cholesterol, HDL, LDL, and triglycerides; and systolic and diastolic blood pressure. Regression analyses all controlled for demographics at step 1 (age, sex, education, marital status, and physical activity). The independent variables added at step 2 were risk awareness, expectancy, intention, planning, and self-efficacy.

The second aim of the study was to determine the effectiveness of a self-directed HAPA-based healthy eating intervention. Prior to assessing intervention effects, pretreatment differences between completers and noncompleters and between the active treatment and control groups were examined, using independent samples $t$-tests and chisquare analyses for continuous and categorical variables, respectively. Intervention effects were assessed using a series of $2 \times 2$ mixed model analyses of variance, with intervention condition (active treatment vs control) as the betweenparticipant effect and time (baseline vs postintervention) as the within-participant effect. Paired samples $t$-tests were subsequently used to explore significant interaction effects. Dependent variables were the aforementioned health outcomes and HAPA variables. The regression variables were checked for multicollinearity, and the tolerance values were considered acceptable. ${ }^{32}$

\section{Results Descriptive statistics}

In all, 77 of the 87 initial participants completed the study (39 in intervention group and 38 in the control group). All participants were Caucasian, with a mean age of 68 years. Most were married (71\%), had completed high school education or above (98\%), and were currently nonsmokers (95\%). The average time since diagnosis of T2D was 10.14 years ( $\mathrm{SD}=7.36$ years, range $=1-45$ years $).$ On average, participants' levels of physical activity were below the recommended level; however, they also generally had better dietary control and healthier eating compared with the general population. ${ }^{24}$ Mean BMI was $31.4 \mathrm{~kg} / \mathrm{m}^{2}(\mathrm{SD}=5.5)$, and on average, both males $(\mathrm{M}=113.4 \mathrm{~cm}, \mathrm{SD}=11.7 \mathrm{~cm})$ and females $(\mathrm{M}=104.7 \mathrm{~cm}, \mathrm{SD}=14.7 \mathrm{~cm})$ had higher than recommended 
waist circumferences. Regarding glycemic management, participants generally reached the target of $7 \mathrm{mmol} / \mathrm{L}$ or less, with an average of $6.7 \mathrm{mmol} / \mathrm{L}(\mathrm{SD}=0.8 \mathrm{mmol} / \mathrm{L})$, equivalent to $5.8 \%$ or $40 \mathrm{mmol} / \mathrm{mol}$. For blood pressure, on average this sample was above the recommended upper level for systolic blood pressure $(\mathrm{M}=141.5 \mathrm{~mm} / \mathrm{Hg}$, $\mathrm{SD}=20.2 \mathrm{~mm} / \mathrm{Hg}$ ) but below for diastolic blood pressure $(\mathrm{M}=77.4 \mathrm{~mm} / \mathrm{Hg}, \mathrm{SD}=11.2 \mathrm{~mm} / \mathrm{Hg})$.

There were no differences between intervention completers and those who dropped out on any of the demographic (eg, age, sex, marital status), control (time since diagnosis, exercise level), or primary outcome variables (dietary behavior, health measures, HAPA variables) as measured at baseline, except that completers reported a lower level of risk awareness $\left(t_{(80)}=2.46, P<0.05\right)$ than noncompleters. The control group were, on average, 8.5 years older than the intervention group $\left(t_{(85)}=3.67, P<0.01\right)$, and the intervention group recorded a significantly higher blood glucose level $(\mathrm{M}=6.9 \mathrm{mmol} / \mathrm{L}[6.2 \%$ or $42 \mathrm{mmol} / \mathrm{mol}])$, compared with the control group $(\mathrm{M}=6.5 \mathrm{mmol} / \mathrm{L}[5.7 \%$ or $39 \mathrm{mmol} / \mathrm{mol}])\left(t_{(81)}=2.14, P<0.05\right)$. No other significant between-group differences were found on any demographic information or baseline variables (all $P>0.05$ ).

\section{Predictive results}

At baseline, there were significant and positive correlations between all HAPA variables, except between planning and risk awareness (Table 1). Healthy eating was not associated with the HAPA variables.

The HAPA variables made a significant contribution to explaining BMI, accounting for $16.6 \%$ of additional variance (see Table 2). Risk awareness and recovery selfefficacy explained independent variance in BMI. Although the HAPA step in the regression did not significantly improve prediction for the other outcomes, some individual HAPA variables explained independent variance: risk awareness explained independent variance in systolic blood pressure; task self-efficacy explained independent variance in diabetes distress; coping self-efficacy explained independent variance in blood glucose level as well as HDL cholesterol; intention explained independent variance in diastolic blood pressure; and intention explained independent variance in HDL cholesterol.

The overall model (including demographics and HAPA variables) was significant for BMI, waist circumference, systolic blood pressure, general cholesterol, and HDL cholesterol, with the greatest proportion of variance explained in BMI ( $40.3 \%$ of variance).

\section{Intervention results}

The repeated measures analyses revealed no significant main effects of time (baseline vs postintervention), treatment condition (active treatment vs control), or time $\times$ condition interaction effects for the majority of measured variables - intention, expectancy, action planning, risk awareness (severity), self-efficacy (task, coping, recovery), healthy eating, diabetes-related distress, BMI, waist circumference, glycemic control, lipids (total cholesterol, HDL, LDL, and triglycerides), and systolic or diastolic blood pressure (all $P>0.05$; see Table 3).

Significant main effects of time were observed for absolute $\left(F_{1,74}=4.026, P=0.048\right)$ and relative risk awareness $\left(F_{1,74}=4.136, P=0.046\right)$, such that perceptions of risk were reduced from baseline to postintervention regardless of treatment condition. A significant main effect of time was also observed for healthy eating $\left(F_{1,74}=5.315, P=0.024\right)$, such that across-conditions scores on the DGI increased from baseline to postintervention. Finally, a significant time $\times$ condition interaction effect was observed for coping planning $\left(F_{1,74}=4.120, P=0.046\right)$. Paired samples $t$-tests indicated that the control condition improved from baseline to postintervention $\left(t_{37}=-3.128, P=0.003\right.$ ), whereas the active treatment condition remained unchanged from baseline $(P>0.05)$.

Table I Correlation between baseline HAPA variables and healthy eating

\begin{tabular}{|c|c|c|c|c|c|c|c|}
\hline & Expectancy & Intention & SE task & SE coping & SE recovery & Planning & Healthy eating \\
\hline Risk & $0.505 * *$ & 0.406 ** & $0.355^{* *}$ & $0.260 *$ & $0.299 * *$ & 0.159 & 0.120 \\
\hline Expectancy & & $0.514 * *$ & $0.535^{* *}$ & $0.432^{* *}$ & $0.385^{* *}$ & $0.272^{*}$ & 0.102 \\
\hline Intention & & & $0.700^{* *}$ & $0.648^{* *}$ & $0.424 * *$ & $0.458 * *$ & -0.014 \\
\hline SE task & & & & $0.808^{* *}$ & $0.56 \mathrm{I} * *$ & $0.520^{* *}$ & 0.097 \\
\hline SE coping & & & & & $0.615^{* *}$ & $0.577^{* *}$ & 0.142 \\
\hline SE recovery & & & & & & $0.415^{* *}$ & 0.134 \\
\hline Planning & & & & & & & 0.191 \\
\hline
\end{tabular}

Notes: $* P<0.05 ; * * P<0.01$.

Abbreviations: SE, self-efficacy; HAPA, health action process approach. 
Table 2 Hierarchical regressions predicting health outcomes at follow up, from baseline control and HAPA variables

\begin{tabular}{|c|c|c|c|c|c|c|c|c|c|c|c|}
\hline & \multirow{3}{*}{$\begin{array}{l}\text { Diabetes } \\
\text { distress }\end{array}$} & \multirow{2}{*}{$\begin{array}{l}\text { Healthy } \\
\text { eating } \\
\end{array}$} & \multirow{3}{*}{$\frac{\mathrm{BMI}}{\beta}$} & \multirow{3}{*}{$\frac{\text { Waist }}{\beta}$} & \multirow{3}{*}{$\begin{array}{l}\begin{array}{l}\text { Blood } \\
\text { glucose }\end{array} \\
\beta\end{array}$} & \multirow{3}{*}{$\begin{array}{l}\text { Systolic } \\
\text { BP } \\
\beta\end{array}$} & \multirow{3}{*}{$\begin{array}{l}\text { Diastolic } \\
\text { BP } \\
\beta\end{array}$} & \multirow{3}{*}{$\frac{\text { Cholesterol }}{\beta}$} & \multirow{3}{*}{$\frac{\text { Triglycerides }}{\beta}$} & \multirow{3}{*}{$\frac{\mathrm{HDL}}{\beta}$} & \multirow{3}{*}{$\frac{\mathrm{LDL}}{\beta}$} \\
\hline & & & & & & & & & & & \\
\hline & & $\beta$ & & & & & & & & & \\
\hline \multicolumn{12}{|c|}{ Step I: controls ${ }^{\mathrm{a}}$} \\
\hline$\Delta R^{2}$ & 0.064 & 0.020 & $0.237^{* *}$ & $0.274 * *$ & 0.125 & 0.141 & 0.137 & $0.188^{*}$ & 0.072 & $0.253 * *$ & 0.080 \\
\hline Age & -0.133 & 0.062 & $-0.34 I^{*}$ & -0.229 & $-0.295^{*}$ & -0.038 & $-0.365 * *$ & -0.114 & -0.172 & 0.256 & -0.254 \\
\hline Sex & 0.059 & 0.081 & -0.012 & $-0.419 * *$ & -0.059 & $-0.299 *$ & -0.048 & $0.369 * *$ & 0.069 & $0.424 * *$ & 0.193 \\
\hline Education & -0.120 & -0.039 & -0.107 & -0.165 & -0.083 & -0.166 & -0.019 & 0.016 & -0.015 & -0.012 & 0.013 \\
\hline Marital status & 0.077 & 0.017 & $-0.236 *$ & -0.194 & 0.232 & 0.120 & 0.195 & $-0.250 *$ & -0.054 & -0.090 & -0.133 \\
\hline Exercise & 0.051 & -0.035 & $-0.287^{*}$ & $-0.336 * *$ & -0.167 & $-0.268^{*}$ & -0.208 & 0.033 & $-0.302^{*}$ & 0.193 & 0.095 \\
\hline \multicolumn{12}{|c|}{ Step 2: HAPA } \\
\hline$\Delta R^{2}$ & 0.167 & 0.126 & $0.166^{*}$ & 0.073 & 0.085 & 0.151 & 0.122 & 0.094 & 0.084 & 0.116 & 0.126 \\
\hline Risk & 0.199 & 0.238 & $0.308^{*}$ & 0.221 & 0.155 & $0.365 * *$ & 0.149 & -0.161 & -0.065 & -0.102 & -0.095 \\
\hline Expectancy & 0.301 & -0.221 & -0.044 & -0.179 & -0.076 & -0.120 & 0.097 & -0.235 & -0.065 & -0.025 & -0.267 \\
\hline Intention & 0.036 & 0.176 & 0.247 & 0.332 & -0.300 & -0.251 & $-0.433^{*}$ & 0.207 & 0.264 & -0.034 & -0.069 \\
\hline SE task & $-0.632 * *$ & -0.237 & -0.300 & -0.098 & -0.045 & 0.137 & -0.062 & 0.054 & -0.374 & 0.330 & 0.173 \\
\hline SE cope & 0.322 & -0.086 & 0.266 & -0.016 & $0.470 *$ & 0.016 & 0.368 & -0.104 & 0.133 & $-0.492 *$ & 0.055 \\
\hline SE recovery & 0.091 & 0.252 & $0.348^{*}$ & -0.117 & -0.106 & -0.029 & -0.181 & 0.112 & -0.105 & $0.36 I^{*}$ & 0.061 \\
\hline Planning & -0.115 & 0.150 & 0.135 & -0.049 & -0.053 & 0.219 & 0.244 & 0.110 & $<0.001$ & 0.032 & 0.226 \\
\hline Total $R^{2}$ & 0.231 & 0.146 & $0.403^{* *}$ & $0.347^{* *}$ & 0.209 & $0.291 *$ & 0.260 & $0.282^{*}$ & 0.156 & $0.369 * *$ & 0.207 \\
\hline
\end{tabular}

Notes: Standardized regression coefficients $(\beta)$ are from step $2 ; * P<0.05$; $* * P<0.01$. a Control variables were "dummy" coded as follows: sex (male $=0$, female $=1$ ); education (high school or less $=0$, further education $=1$ ), marital status (single $=0$, married $=1$ ).

Abbreviations: BMI, body mass index; BP, blood pressure; Waist, waist circumference; HAPA, health action process approach; SE, self-efficacy; HDL, high density lipoprotein; LDL, low density lipoprotein.

\section{Discussion}

There were two aims to this research, firstly to explore whether the HAPA is a useful model within which to predict health outcomes in individuals with T2D, and secondly, to test the effectiveness of a self-directed, HAPA-based, healthy eating intervention on health outcomes in individuals with T2D.

\section{Predictive findings}

Mixed support was found for the efficacy of the HAPA model in predicting health outcomes among people with T2D. None of the HAPA variables were associated with healthy eating at baseline, nor did they predict healthy eating following the intervention period. However, previous research using the HAPA to investigate healthy eating has supported the model. ${ }^{33,34}$ It is possible that because the current sample had better dietary control and healthier eating than the general population, changes in healthy eating could not be detected, and it may be that the intervention could have worked in a group with less healthy eating. It is also possible that the healthy eating measure used had limitations within this sample, despite having been used with relative success in previous research. ${ }^{24}$ In a review of dietary indexes, Arvaniti and Panagiotakos ${ }^{35}$ noted that the way in which dietary scales classify foods to create a single score can be problematic and oversimplified. Previous research has noted the challenges in accurately measuring food and eating, ${ }^{36}$ and suggest that clear measures are still to be developed. Further research is needed to determine the most appropriate dietary measure to use in this context.

It is worth noting that the HAPA variables were generally correlated with each other. Although the HAPA has not been used to explore healthy eating in those with T2D, the correlations between HAPA variables found in the current study were generally similarly or more highly correlated than in previous studies investigating the HAPA and healthy eating, ${ }^{37}$ thus increasing the strength of the validity of the measures and suggesting that survey implementation characteristics are unlikely to account for the pattern of findings.

Regarding other health outcomes, the overall HAPA model significantly improved the prediction of BMI over demographic and control variables, suggesting that the model may have utility in the domain of weight control. However, the HAPA model did not improve prediction of the other health outcomes. Apart from healthy eating, the outcomes were indicators of physical health, which may be more difficult to predict and change than health behaviors. Given that healthy eating was not predicted in the current study, further exploration of other health behaviors that may form the link between HAPA variables and physical health measures in this context is warranted, which may help to improve the prediction of such health outcomes. Furthermore, given that several analyses were conducted with different outcomes, the scale 
Table 3 ANCOVA analysis of the healthy eating intervention on change in outcome and HAPA variables

\begin{tabular}{lllll}
\hline $\begin{array}{l}\text { Variable } \\
\text { group }\end{array}$ & Variable name & $\boldsymbol{F}$ & $\boldsymbol{P}$ & Partial $\eta^{2}$ \\
\hline Outcome & Diabetes distress & 0.84 & 0.36 & $0.01 \mathrm{I}^{+}$ \\
variables & Healthy eating & 0.04 & 0.84 & 0.001 \\
& BMI & 0.58 & 0.45 & 0.008 \\
& Waist circumference & 1.82 & 0.18 & $0.030^{+}$ \\
& Blood glucose level & 0.26 & 0.61 & 0.004 \\
& Blood pressure, systolic & 0.92 & 0.34 & $0.013^{+}$ \\
& Blood pressure, diastolic & 0.00 & 0.10 & 0.000 \\
& Cholesterol: total & 2.28 & 0.14 & $0.030^{+}$ \\
& Triglycerides & 1.48 & 0.23 & $0.020^{+}$ \\
& HDL ("good cholesterol”) & 0.04 & 0.85 & 0.000 \\
Process & LDL ("bad cholesterol”) & 0.11 & 0.74 & 0.002 \\
variables & Risk & 0.01 & 0.93 & 0.000 \\
& Expectancy & 1.09 & 0.50 & $0.015^{+}$ \\
& Intention & 0.96 & 0.33 & $0.013^{+}$ \\
& Self-efficacy task & 0.99 & 0.32 & $0.014^{+}$ \\
& Self-efficacy coping & 0.25 & 0.62 & 0.003 \\
& Self-efficacy recovery & 1.00 & 0.83 & 0.001 \\
& Planning & 0.98 & 0.24 & $0.020^{+}$ \\
\hline
\end{tabular}

Notes: Age and baseline levels of outcome variables were controlled for; ${ }^{+}$small effect size (partial $\eta^{2}=0.010-0.060$ ).

Abbreviations: ANCOVA, analysis of covariance; BMI, body mass index; HAPA, health action process approach.

of the results that were significant needs to be considered in context, suggesting that overall, the HAPA model was of somewhat limited utility in predicting health outcomes in this population.

Nonetheless, some of the HAPA variables were individually relevant in predicting health outcomes, with the greatest findings occurring within self-efficacy. That task self-efficacy predicted diabetes distress suggests that those who have confidence in being able to start a healthy routine feel less burdened by their illness. Coping self-efficacy predicted both blood glucose level and HDL cholesterol, whilst recovery self-efficacy also predicted HDL cholesterol as well as BMI. These finding reflects the relative strength of the construct of self-efficacy reported within the literature ${ }^{14,38,39}$ and suggest that feeling confident and able to engage in healthy behaviors is important for many diabetes health-related outcomes. That different aspects of self-efficacy predicted different outcomes lends credence to the belief that a more nuanced exploration of self-efficacy may improve the understanding of health behaviors and health outcomes, and may assist in designing specific interventions for certain health outcomes. However, this finding may also indicate that those who are healthier have a greater sense of self-efficacy, and thus the direction of the relationships between specific components of self-efficacy and health outcomes need to be experimentally tested within this population.

Risk awareness was also an important predictor for BMI and systolic blood pressure. This is in contrast to previous research that found risk awareness to be a less important construct within the HAPA, even amongst individuals with chronic health problems. ${ }^{39}$ Given that those with greater risk perceptions had higher BMIs in the current study, perhaps a logical explanation is that those who are more overweight accurately report their higher health risks. However, this needs to be specifically tested. Further, it may be that completers and noncompleters apparently differed on risk awareness because those with low risk awareness had already withdrawn from the study.

That intention was only predictive of systolic blood pressure was surprising as previous research has shown intention to be an important determinant of health behavior, ${ }^{40,41}$ and health behavior has subsequently been linked to health outcomes. ${ }^{42,43}$ One explanation for the lack of prediction of health outcomes is that intentions are not always acted on, a trend that has been termed the "intention-behavior gap". ${ }^{40}$ As such, if intentions to eat healthily (or engage in other health behaviors) are not acted upon consistently, it is unlikely that flow-on effects to physical health outcomes would be observed, particularly for longer-term outcomes, such as blood glucose levels and BMI. Within the HAPA, planning is proposed to mediate the intention-behavior gap; however, as planning was not associated with any of the health outcomes or dietary behavior in the current study, further exploration of alternative ways of closing the intention-behavior gap is warranted. Recent research has shown that within individuals with other chronic diseases, such as celiac disease, the presence of depressive symptoms decreases the likelihood of translating intention into behavior; ${ }^{44}$ whether this also applies in people with T2D would be worth investigating.

\section{Intervention findings}

Against predictions, the HAPA intervention was not supported in the current study as no significant interaction effects (with the exception of coping planning, which was in the opposite direction to expectations) were found on HAPA variables or health outcome variables between the treatment groups. This is in contrast to previous research, in which preliminary evidence of the effectiveness of very lowintensity interventions to manage food intake, in overweight women ${ }^{45}$ and in individuals at risk of T2D, ${ }^{19}$ has been found. However, in individuals with T2D, the intervention evidence is mixed. Support has been found for improvement in 
proximal predictors of diabetes self-management, including self-efficacy and healthy eating in studies that involved fewer than 15 hours of individual or group contact. ${ }^{46,47}$ Despite this, the effectiveness of a low-intensity lifestyle counseling intervention on self-management was not supported in a randomized controlled trial. ${ }^{48}$ Furthermore, these lowintensity interventions have not used the HAPA, and therefore comparisons with the previous research are limited. One low-intensity HAPA intervention that has been studied was not successful in increasing flu vaccination uptake ${ }^{49}$ and may suggest limitations of the utility of this model within a low-intensity approach.

It was interesting to note that despite no significant effects on HAPA variables, improvements in healthy eating across the course of the intervention for participants, in both conditions, were observed. This may reflect a "mere measurement effect" - a phenomenon found in health psychology research, whereby being merely asked about health attitudes and behaviors can result in behavior change. ${ }^{50,51}$ That this finding was more pronounced than the HAPA specific intervention effects suggests that further research into the utility of the mere measurement effect within the context of health behavior interventions for T2D is warranted.

It may be that the HAPA is not an effective model for targeting healthy eating for people with T2D. When considering the predictive findings alongside the intervention findings, it appears that HAPA variables are not associated with key outcomes, such as healthy eating. Alternative models could therefore be explored as the basis for health behavior interventions for people with T2D. An alternative explanation for the lack of intervention effectiveness is that the intervention may have been too brief to create meaningful changes in the independent variables (HAPA and diabetes-related health), despite improvements in healthy eating. Although increasing the intensity or length of the intervention may lead to greater success, the purpose of the current research was to explore ways to create brief low-cost interventions that may be more accessible to a wider population. Therefore, alternative ways to create brief, low-cost interventions need to be explored. Previous research has found brief, non-face-to-face interventions to be effective in improving dietary behavior and cholesterol in individuals with diabetes, up to 12 months later, ${ }^{52}$ suggesting that change can be effectively brought about within this population following brief interventions. Furthermore, the limited predictive ability of the HAPA model suggests that further research targeting alternative mechanisms is needed before judgment can be made about whether brief interventions can be effective.
Given that support has been found for brief, low-intensity interventions ${ }^{15}$ and that there is preliminary evidence to support the use of the HAPA model to change behavior, ${ }^{18,19}$ it appears that the lack of effectiveness of the current intervention may be due to the interaction between population, model, and intervention. Those with T2D may face additional challenges to those in the general population that make it more difficult for these individuals to improve health behaviors, such as healthy eating. As such, interventions may pose a particular challenge within this group. Future research could explore these potential challenges and attempt to develop effective and accessible interventions, perhaps drawing on interventions that are found to be more effective in other populations with chronic health difficulties. ${ }^{20,28}$

An additional finding worth considering is that those who completed the intervention generally reported a lower level of risk awareness than did those who did not complete the intervention, although no other differences between these groups were found. Those with lower levels of risk perception may feel less threatened to engage in the intervention. Alternatively, risk perceptions may reflect actual risk such that those who are generally healthier may also be more motivated to complete the intervention. These risk hypotheses have been considered within the literature, ${ }^{53}$ although they have not been explicitly compared within the diabetes research. These would be worth investigating so that interventions can be better targeted to those for whom there would be most benefit.

\section{Limitations}

It is worth noting that although the intervention was designed to target the three different stages of health behavior change within the HAPA model (preintention, intention, and action), it is possible that participants completed these stages of the intervention when they were not themselves at this stage, thus potentially creating a mismatch between the needs of the individual and the program. Further studies could try to tailor stage-based interventions, although this approach may be more resource intensive. Furthermore, the follow-up length may have been too short to identify changes in physical health outcomes as previous studies have used longer lengths. ${ }^{17,19}$ In addition, that the HAPA variables did not predict healthy eating may reflect a limitation of the healthy eating measure. Further research is needed to compare and contrast the existing measures in order to identify the best measures to use within health behavior research. Low response rates may also have influenced the results and may limit generalizability of these findings to individuals with T2D as a whole. Finally, whilst "mere measurement effects" were found across 
groups, it is also possible that these could have occurred between groups. Future researchers could consider using a Solomon four-group design to account for such effects.

\section{Implications and future directions}

The self-management intervention employed in the current study was ineffective, which when taken together with the predictive results, suggests that the HAPA may be a limited framework within which to explore ways to improve health of those with T2D; however, this may reflect a limitation of the intervention and dietary measures rather than the model per se. Therefore, as effective and low-cost health interventions for individuals with T2D are still needed, different approaches should be explored. Given that the current intervention targeted diet alone, future interventions could target other single or combinations of health behaviors that are relevant to those with T2D. For example, a recent intervention targeting healthy eating and physical activity in older adults with T2D and cardiovascular disease, based on the theory of planned behavior, found improvements in physical activity but not in healthy eating, suggesting that exercise may make a more effective behavioral and health target within this population. ${ }^{54}$

\section{Conclusion}

This study explored 1) whether HAPA variables were associated with health outcomes, and 2) whether a low-cost, HAPA-based self-management intervention could improve healthy eating and physical health outcomes in individuals with T2D. The model had some success in predicting health outcomes, although this was limited, and healthy eating was not predicted. The relatively low predictive ability of HAPA shown in this and other studies ${ }^{13,38}$ highlights the need for alternative ways to explain and improve health in those with T2D. That the intervention was also not effective suggests that further exploration of ways in which to improve health outcomes in those with T2D is needed, potentially making use of other health models or other methods of intervention implementation.

\section{Disclosure}

The authors report no conflicts of interest in this work.

\section{References}

1. Australian Institute of Health and Welfare. Diabetes: Australian Facts 2008. Canberra: Australian Institute of Health and Welfare; 2008. Available from: http://www.aihw.gov.au/publication-detail/?id=6442468075. Accessed August 20, 2013.

2. Barr ELM, Magliano DJ, Zimmet PZ, et al. AusDiab 2005. The Australian Diabetes, Obesity and Lifestyle Study. Tracking the Accelerating Epidemic: Its Causes and Outcomes. Melbourne: International Diabetes Institute; 2006.
3. Wild S, Roglic G, Green A, Sicree R, King H. Global prevalence of diabetes: estimates for the year 2000 and projections for 2030. Diabetes Care. 2004;27(5):1047-1053.

4. World Health Organization Diabetes. Health topics: Diabetes. World Health Organization; [cited May 2013]. Available from: http://www. who.int/topics/diabetes_mellitus/en/. Accessed August 20, 2014.

5. Klein S, Sheard NF, Pi-Sunyer X, et al; American Diabetes Association; North American Association for the Study of Obesity; American Society for Clinical Nutrition. Weight management through lifestyle modification for the prevention and management of type 2 diabetes: rationale and strategies. A statement of the American Diabetes Association, the North American Association for the Study of Obesity, and the American Society for Clinical Nutrition. Diabetes Care. 2004;27(8):2067-2073.

6. National Collaborating Centre for Chronic Conditions, National Institute for Health and Clinical Excellence. Type 2 Diabetes: National Clinical Guideline for Management in Primary and Secondary Care (Update). London: Royal College of Physicians; 2008.

7. Diabetes Australia. Diabetes Management in General Practice: Guidelines for Type 2 Diabetes. Gorokan: Pinnacle Print Management; 2009. Available from: http://www.diabetesaustralia.com.au/Documents/ DA/What's New/12.10.02 Diabetes Management in General Practice. pdf. Accessed August 20, 2014.

8. Conner M, Norman P. Predicting health behaviour: A social cognition approach. In: Conner M, Norman P, editors. Predicting Health Behaviour: Research and Practice with Social Cognition Models. Buckingham: Open University Press; 2005;1-27.

9. Schwarzer R. Self-efficacy in the adoption and maintenance of health behaviours: Theoretical approaches and a new model. In: Schwarzer R, editor. Self-Efficacy: Thought Control of Action. Washington: Taylor and Francis; 1992;217-243.

10. Bandura A. Cultivate self-efficacy for personal and organizational effectiveness. In: Locke EA, editor. The Blackwell Handbook of Principles of Organizational Behavior. New York: Wiley-Blackwell; 2000:120-136.

11. Gough SC, Kragh N, Ploug UJ, Hammer M. Impact of obesity and type 2 diabetes on health-related quality of life in the general population in England. Diabetes Metab Syndr Obes. 2009;2:179-184.

12. Gollwitzer PM. Implementation intentions: Strong effects of simple plans. Am Psychol. 1999;54(7):493-503.

13. Renner B, Kwon S, Yang BH, et al. Social-cognitive predictors of dietary behaviors in South Korean men and women. Int J Behav Med. 2008;15(1):4-13.

14. Schwarzer R, Renner B. Social-cognitive predictors of health behavior: action self-efficacy and coping self-efficacy. Health Psychol. 2000;19(5):487-495.

15. Parschau L, Barz M, Richert J, Knoll N, Lippke S, Schwarzer R. Physical activity among adults with obesity: testing the Health Action Process Approach. Rehabil Psychol. 2014;59(1):42-49.

16. Lippke S, Plotnikoff RC. Testing two principles of the Health Action Process Approach in individuals with type 2 diabetes. Health Psychol. 2014;33(1):77-84.

17. Nation M, Crusto C, Wandersman A, et al. What works in prevention: Principles of effective prevention programs. Am Psychol. 2003;58(6-7):449-456.

18. Ströbl V, Knisel W, Landgraf U, Faller H. A combined planning and telephone aftercare intervention for obese patients: effects on physical activity and body weight after one year. J Rehabil Med. 2013;45(2): 198-205.

19. Laatikainen T, Dunbar JA, Chapman A, et al. Prevention of type 2 diabetes by lifestyle intervention in an Australian primary health care setting: Greater Green Triangle (GGT) Diabetes Prevention Project. BMC Public Health. 2007; 7:249.

20. Warsi A, Wang PS, LaValley MP, Avorn J, Solomon DH. Selfmanagement education programs in chronic disease: A systematic review and methodological critique of the literature. Archives of Internal Medicine. 2004;164(15):1641-1649.

21. Dalle Grave R, Calugi S, Centis E, Marzocchi R, El Ghoch M, Marchesini G. Lifestyle modification in the management of the metabolic syndrome: achievements and challenges. Diabetes Metab Syndr Obes. 2010;3:373-385. 
22. Avery L, Flynn D, van Wersch A, Sniehotta FF, Trenell MI. Changing physical activity behavior in type 2 diabetes: a systematic review and meta-analysis of behavioral interventions. Diabetes Care. 2012;35(12): 2681-2689.

23. fu-berlin.de [homepage on the Internet]. Health Action Process Approach (HAPA). Freie Universität Berlin; 2009 [updated December 11, 2010; cited Mar 2009]. Available from: http://userpage.fu-berlin.de/ health/ hapa.htm. Accessed August 20, 2014.

24. Sniehotta FF, Scholz U, Schwarzer R. Bridging the intention-behaviour gap: Planning, self-efficacy, and action control in the adoption and maintenance of physical exercise. Psychol Health. 2005;20(2):143-160.

25. McNaughton SA, Ball K, Crawford D, Mishra GD. An index of diet and eating patterns is a valid measure of diet quality in an Australian population. J Nutr. 2008;138(1):86-93.

26. Kellett E, Smith A, Schmerlaib Y. The Australian Guide to Healthy Eating. Canberra: Commonwealth Department of Health and Family Services. 1998.

27. Polonsky WH, Fisher L, Earles J, et al. Assessing psychosocial distress in diabetes: development of the diabetes distress scale. Diabetes Care. 2005;28(3):626-631.

28. National Institute for Health and Clinical Excellence. Clinical Guideline 43: Obesity: Guidance on the Prevention, Identification, Assessment and Management of Overweight and Obesity in Adults and Children. London: National Institute for Health and Clinical Excellence; 2006.

29. Lorig KR, Ritter P, Stewart AL, et al. Chronic disease self-management program: 2-year health status and health care utilization outcomes. Med Care. 2001;39(11):1217-1223.

30. Thoolen B, de Ridder D, Bensing J, Gorter K, Rutten G. Beyond Good Intentions: the development and evaluation of a proactive selfmanagement course for patients recently diagnosed with type 2 diabetes. Health Educ Res. 2008;23(1):53-61.

31. Rollnick S, Miller WR, Butler CC. Motivational Interviewing in Health Care: Helping Patients Change Behavior. New York, NY: The Guilford Press; 2007.

32. Yesavage JA, Kinoshita LM, Noda A, et al. Effects of body mass indexrelated disorders on cognition: preliminary results. Diabetes Metab Syndr Obes. 2014;7:145-151.

33. Wiedemann AU, Lippke S, Reuter T, Schüz B, Ziegelmann JP, Schwarzer R. Prediction of stage transitions in fruit and vegetable intake. Health Educ Res. 2009;24(4):596-607.

34. Scholz U, Ochsner S, Hornung R, Knoll N. Does social support really help to eat a low-fat diet? Main effects and gender differences of received social support within the Health Action Process Approach. Appl Psychol Health Well Being. 2013;5(2):270-290.

35. Arvaniti F, Panagiotakos DB. Healthy indexes in public health practice and research: a review. Crit Rev Food Sci Nutr. 2008;48(4):317-327.

36. Glanz K. Measuring food environments: a historical perspective. Am J Prev Med. 2009;36(Suppl 4):S93-S98.

37. Schwarzer R, Schuz B, Ziegelmann JP, Lippke S, Luszczynska A, Scholz U. Adoption and maintenance of four health behaviors: theoryguided longitudinal studies on dental flossing, seat belt use, dietary behavior, and physical activity. Ann Behav Med. 2007;33(2):156-166.

38. Schwarzer R, Luszczynska A, Ziegelmann JP, Scholz U, Lippke S. Socialcognitive predictors of physical exercise adherence: three longitudinal studies in rehabilitation. Health Psychol. 2008;27(Suppl 1):S54-S63.
39. Schwarzer R, Luszczynska A. How to overcome health-compromising behaviors. The health action process approach. Eur Psychol. 2008;13(2): $141-151$.

40. Sheeran P. Intention-behavior relations: A conceptual and empirical review. Eur Rev Soc Psychol. 2002;12(1):1-36.

41. Schwarzer R, Fuchs R. Self-efficacy and health behaviours. In: Conner M, Norman P, editors. Predicting Health Behaviour: Research and Practice with Social Cognition Models. Buckingham: Open University Press; 1996:163-196.

42. diabetesaustralia.com.au. Type 2 diabetes. Diabetes Australia; 2013 [updated December 19, 2013; cited Jan 2014]. Available from: http:// www.diabetesaustralia.com.au/Understanding-Diabetes/What-isDiabetes/Type-2-Diabetes/. Accessed August 20, 2014.

43. Alberti KG, Zimmet P, Shaw J. International Diabetes Federation: a consensus on type 2 diabetes prevention. Diabet Med. 2007;24(5) $451-463$.

44. Sainsbury K, Mullan B. Measuring beliefs about gluten free diet adherence in adult coeliac disease using the theory of planned behaviour. Appetite. 2011;56(2):476-483.

45. Lombard CB, Deeks AA, Ball K, Jolley D, Teede HJ. Weight, physical activity and dietary behavior change in young mothers: short term results of the HeLP-her cluster randomized controlled trial. Nutr J. 2009;8:17.

46. Wattana C, Srisuphan W, Pothiban L, Upchurch SL. Effects of a diabetes self-management program on glycemic control, coronary heart disease risk, and quality of life among Thai patients with type 2 diabetes. Nurs Health Sci. 2007;9(2):135-141.

47. Young RJ, Taylor J, Friede T, et al. Pro-active call center treatment support (PACCTS) to improve glucose control in type 2 diabetes: a randomized controlled trial. Diabetes Care. 2005;28(2):278-282.

48. Shibayama T, Kobayashi K, Takano A, Kadowaki T, Kazuma K. Effectiveness of lifestyle counseling by certified expert nurse of Japan for non-insulin-treated diabetic outpatients: a 1-year randomized controlled trial. Diabetes Res Clin Pract. 2007;76(2):265-268.

49. Payaprom Y, Bennett P, Alabaster E, Tantipong H. Using the Health Action Process Approach and implementation intentions to increase flu vaccine uptake in high risk Thai individuals: a controlled before-after trial. Health Psychol. 2011;30(4):492-500.

50. Godin G, Sheeran P, Conner M, Germain M. Asking questions changes behavior: mere measurement effects on frequency of blood donation. Health Psychol. 2008;27(2):179-184.

51. Morwitz VG, Fitzsimons GJ. The mere-measurement effect: Why does measuring intentions change actual behavior? J Consum Psychol. 2004;14(1-2):64-74.

52. Glasgow RE, La Chance PA, Toobert DJ, Brown J, Hampson SE, Riddle MC. Long-term effects and costs of brief behavioural dietary intervention for patients with diabetes delivered from the medical office. Patient Educ Couns. 1997;32(3):175-184.

53. Brewer NT, Weinstein ND, Cuite CL, Herrington JE Jr. Risk perceptions and their relation to risk behavior. Ann Behav Med. 2004;27(2) $125-130$.

54. White KM, Terry DJ, Troup C, et al. An extended theory of planned behavior intervention for older adults with type 2 diabetes and cardiovascular disease. J Aging Phys Act. 2012;20(3):281-299.

Diabetes, Metabolic Syndrome and Obesity: Targets and Therapy

\section{Publish your work in this journal}

Diabetes, Metabolic Syndrome and Obesity: Targets and Therapy is an international, peer-reviewed open-access journal committed to the rapid publication of the latest laboratory and clinical findings in the fields of diabetes, metabolic syndrome and obesity research Original research, review, case reports, hypothesis formation, expert opinion and commentaries are all considered for publication. The manuscript management system is completely online and includes a very quick and fair peer-review system, which is all easy to use. Visi http://www.dovepress.com/testimonials.php to read real quotes from published authors. 